\title{
Severe Acute Heart Failure - Experimental Model With Very Low Mortality
}

\author{
S. LACKO ${ }^{1}$, M. MLČEK ${ }^{1}$, P. HÁLA ${ }^{1,2}$, M. POPKOVÁ ${ }^{1}$, D. JANÁK ${ }^{1,3}$, M. HRACHOVINA ${ }^{1}$, \\ J. KUDLIČKA ${ }^{1}$, V. HRACHOVINA ${ }^{1}$, P. OŠŤÁDAL ${ }^{2}$, O. KITTNAR ${ }^{1}$ \\ ${ }^{1}$ Department of Physiology, First Faculty of Medicine, Charles University, Prague, Czech Republic, \\ ${ }^{2}$ Department of Cardiology, Na Homolce Hospital, Prague, Czech Republic, ${ }^{3}$ Department of \\ Cardiovascular Surgery, Second Faculty of Medicine, Charles University, Prague, Czech Republic
}

Received October 7, 2017

Accepted March 2, 2018

On-line May 10, 2018

\section{Summary}

The growth in the experimental research of facilities to support extracorporeal circulation requires the further development of models of acute heart failure that can be well controlled and reproduced. Two types of acute heart failure were examined in domestic pigs (Sus scrofa domestica): a hypoxic model $(n=5)$ with continuous perfusion of the left coronary artery by hypoxic deoxygenated blood and ischemic model $(n=9)$ with proximal closure of the left coronary artery and controlled hypoperfusion behind the closure. The aim was a severe, stable heart pump failure defined by hemodynamic parameters changes: a) decrease in cardiac output by at least $50 \%$; b) decrease in mixed venous blood saturation to under $60 \%$; c) left ventricular ejection fraction below $25 \%$; and d) decrease in flow via the carotid arteries at least $50 \%$. Acute heart failure developed in the first group in one animal with no acute mortality and in the second group in 8 animals with no acute mortality. In the case of ischemic model the cardiac output fell from $6.70 \pm 0.89 \mathrm{l} / \mathrm{min}$ to $2.89 \pm 0.75 \mathrm{l} / \mathrm{min}$. The saturation of the mixed venous blood decreased from $83 \pm 2 \%$ to $58 \pm 8 \%$. The left ventricular ejection fraction decreased from $50 \pm 8 \%$ to $19 \pm 2 \%$. The flow via the carotid arteries decreased from $337 \pm 78 \mathrm{ml} / \mathrm{min}$ to $136 \pm 59 \mathrm{ml} / \mathrm{min}$ ( $P \leq 0.001$ for all comparisons). The proposed ischemic model is not burdened with acute mortality in the development of heart failure and is suitable for further use in experimental research into extracorporeal circulatory support.

\section{Key words}

Animal model $\bullet$ Acute heart failure - Hemodynamics • Cardiac output

\section{Corresponding author}

O. Kittnar, Department of Physiology, First Faculty of Medicine, Charles University, Albertov 5, 128 00, Prague 2, Czech Republic. E-mail: Otomar.Kittnar@staff.cuni.cz

\section{Introduction}

The rapid adoption of extracorporeal circulatory support in acute medicine is linked with the need for further experimental research into these devices. The demand for development of appropriate biomodels is increasing, whereby their properties should meet the basic conditions and requirements for their further use. These properties include: good adjustability of failure degree to severe failure, reproducibility, relatively simple and quick availability, and low acute mortality.

Refractory cardiogenic shock progressing despite the standard available treatment or cardiac arrest making blood flow impossible to be restored using standard resuscitation techniques represent critical circulatory failure with predicted mortality approaching 100 \% (Soleimani and Pae 2012). Experimental results suggest that despite severe metabolic and hemodynamic deterioration during prolonged cardiac arrest, restitution of adequate circulation and oxygenation can rapidly revert otherwise fatal prognosis, thus carrying a potential for dramatic improvement in survival rate (Mlcek et al. 2012).

For the rapid transfer of knowledge to clinical medicine a large porcine biomodel is the most 
appropriate, in particular when taking into consideration its reasonable physiological and anatomical similarities with human being as well as the size of the instrumentation (catheters and cannulas) and extracorporeal support (Crick et al. 1998, Hughes 1986). The model has to enable comprehensive measurement of required parameters under well-defined and controlled conditions that could not be possible in clinical medicine. Undoubtedly, it would be ethically unacceptable to change the settings of the supporting system for the purpose of monitoring its tolerance by a patient under clinical situation.

At present, there is a large number of experimental models of acute and chronic heart failure (HF) based on various techniques including balloon obstruction, ligation or embolization of the coronary arteries. Unfortunately these models are subject to a high degree of acute arrest with considerably high mortality (Kim et al. 2011, Varga-Szemes et al. 2012).

Therefore, the aim of this study was to develop and prepare a biomodel for acute cardiogenic shock that would be suitable for further experimental research of extracorporeal methods supporting the failing circulation, specifically in our case for further study of pathophysiological mechanisms of adverse events that could accompany a clinical use of a venoarterial arrangement of ECMO (VA ECMO).

Moreover, this model could also serve to clarify the pathophysiology of serious complications, such as distension of the left-sided heart chambers and subsequent pulmonary edema in the treatment of VA ECMO, their early diagnosis and the proposal of processes with regards to their limitations (Fuhrman et al. 1999, Soleimani and Pae 2012, Ostadal et al. 2015).

In order to achieve these aims two types of models of acute heart failure were examined: a) a hypoxic model with continuous perfusion of the coronary arteries by hypoxic deoxygenated blood; and b) an ischemic model of controlled hypoperfusion of one of the main branches of the left coronary artery: left anterior descending artery (LAD) or left circumflex artery (LCx).

\section{Methods}

The study was realized in the Electrophysiological Experimental Laboratory, Department of Physiology, First Faculty of Medicine, Charles University, where a broad range of knowledge suitable for the performance of the project of the given range was available. The equipment and expertise garnered through projects - models of the heart failure and hemodynamic support - provided the ideal venue for the planned study. Experimental protocol was reviewed and approved by the Institutional Animal Expert Committee of First Faculty of Medicine, Charles University. All animals were treated and cared for in accordance with the Guide for the Care and Use of Laboratory Animals (1985), NIH, Bethesda.

\section{Animal model}

Fourteen healthy female pigs $(45 \pm 4.1 \mathrm{~kg})$ were used. A hypoxic model with continuous perfusion of the left coronary artery by hypoxic deoxygenated blood was performed on 5 animals and an ischemic model with proximal closure of the left coronary artery was realized on 9 animals. The required severe stable heart pump failure was defined by following hemodynamic parameter changes: a) decrease in cardiac output (CO) by at least $50 \%$ accompanied by a decrease in stroke volume (SV) and heart rate (HR) change; b) decrease in mixed venous blood saturation $\left(\mathrm{SvO}_{2}\right)$ to values below $60 \%$, which corresponds to an insufficient oxygen supply to the tissues; c) left ventricular ejection fraction (LVEF) below $25 \%$; and d) decrease in the arterial blood flow in the periphery, in this case via the carotid arteries (CarFlow) by at least $50 \%$.

Following the premedication with midazolame $(0.3 \mathrm{mg} / \mathrm{kg})$ along with a dose of ketamine $(20 \mathrm{mg} / \mathrm{kg})$ i.m., an intravenous entry into the peripheral bloodstream was obtained on the ear and the animal was subsequently transported to the laboratory. An initial bolus dose of propofol $(2 \mathrm{mg} / \mathrm{kg})$ was administered. The animal was pre-oxygenated with $100 \%$ oxygen via a facial mask and after a subsequent orotracheal intubation connected to a mechanical ventilator (Hamilton G5, Hamilton Medical $A G$, Switzerland). Ventilation was performed under the INTELLiVENT mode setting (Adaptive Support Ventilation mode) with the possibility of real time monitoring of pulmonary mechanics and ventilation optimization according to a) airway pressure, b) peripheral blood saturation with oxygen $\left(\mathrm{SpO}_{2}\right)$, and c) $\mathrm{CO}_{2}$ levels at the end of exhalation $\left(\mathrm{etCO}_{2}\right)$. Total intravenous anesthesia was maintained by continuous i.v. administration of propofol $(6-8 \mathrm{mg} / \mathrm{kg} . \mathrm{h})$, midazolame ( 0.1 to $0.2 \mathrm{mg} / \mathrm{kg} . \mathrm{h})$ and morphine $(0.1$ to $0.2 \mathrm{mg} / \mathrm{kg} . \mathrm{h})$. To achieve the central venous pressure (CVP) of $5-6 \mathrm{~mm} \mathrm{Hg}$, a saline was initially administered at the rate of $500 \mathrm{ml} / \mathrm{h}$. The initial bolus dose of heparine 
(100 IU/kg) was followed by the continuous administration of $50 \mathrm{IU} / \mathrm{kg}$.h to keep the activated clotting time (ACT) over $200 \mathrm{~s}$. Throughout the protocol the following interventions were used: a) the ventilation target was set up to keep $\mathrm{SpO}_{2}$ in the range from $95 \%$ to $99 \%$, the tidal volume at $7 \mathrm{ml} / \mathrm{kg}$ and a respiratory rate to keep etCO $\mathrm{C}_{2}$ between 38 and $42 \mathrm{~mm} \mathrm{Hg}$; b) continuous administration of norepinephrine (up to $1.5 \mu \mathrm{g} / \mathrm{kg} . \mathrm{min}$ ) to maintain mean arterial pressure (MAP) above $60 \mathrm{~mm} \mathrm{Hg}$; c) continuous administration of crystalloid in order to maintain CVP at approximately $5 \mathrm{~mm} \mathrm{Hg}$.

\section{Experimental preparation and monitoring}

Following the induction of general anesthesia and connection to artificial lung ventilation, percutaneous catheters with measuring sensors were inserted for the continuous scanning of experimental data. Continuous ECG recording, $\mathrm{SpO}_{2}$ measurements using pulse oximetry, rectal temperature and etCO $\mathrm{C}_{2}$ were monitored using a bedside monitor (LifeScope TR, Nihon Kohden, Japan). A Swan-Ganz pulmonary artery catheter (CCO, Edwards Lifesciences, USA) inserted into the pulmonary artery via femoral vein and inferior vena cava was used for the measurement of pulmonary artery pressure and $\mathrm{SvO}_{2}$. Blood coagulation was regularly monitored using ACT, Hemochron Junior (International Technidyne Corporation, Edison, NJ, USA). A high fidelity PressureVolume conductance catheter (type 5F, 7F VSL, Scisense-Transonic, USA) was used for continuous real-time measurement of pressure and volume changes inside the left ventricle during the cardiac cycle. The catheter was introduced under the fluoroscopic control through the left carotid artery and the aortic valve. Blood gasses and blood oxygen saturation in femoral vein (in the case of hypoxic model just behind the oxygenator) $\left(\mathrm{SfvO}_{2}\right)$ were recorded using the real-time analyzer (CDI 500, Terumo, Japan). Continuous monitoring of $\mathrm{CO}$ during the heart failure development was acquired and analyzed using a PV unit (Scisense Inc. ADVantage PV System, Ontario, Canada) operating in the admittance mode (Raghavan et al. 2004). LVEF was calculated as the ratio of $\mathrm{SV}$ to the end-diastolic volume (EDV) of the left ventricle (LVEF $=\mathrm{SV} / \mathrm{EDV})$, whereby the SV is the difference between the EDV and end-systolic left ventricular volumes (ESV). CO was obtained as HR x $\mathrm{SV}$. Invasive blood pressure measurement was taken using the pigtail catheter inserted through the femoral artery to the aortic arch connected to the fluid-filled probe (Truwave, Edwards Lifesciences, USA) calibrated at the heart level. Continuous volumetric measurement of the flow in the periphery was measured using a Transient Ultrasound Flow Probe (PSB, Transonic, USA) implanted on the exposed right-sided carotid artery. All the measured parameters were sampled at $400 \mathrm{~Hz}$ by Powerlab A/D converter. Data were continuously recorded by Labchart Pro software (AD Instruments, USA).

\section{Acute heart failure induction}

Prior to the heart failure induction the baseline period of no intervention for at least 20 min was warranted.

$\mathrm{HF}$ in the hypoxic model was induced during total intravenous anesthesia by catheter perfusion of the left coronary artery orifice by gradually deoxygenated blood. The venous blood was collected through a catheter placed in the femoral vein and forced by a dedicated pump via a catheter inserted through the radial artery into the orifice of the coronary artery (Fig. 1). The perfusion by venous blood was set to the minimum flow rate of $250 \mathrm{ml} / \mathrm{min}$ in order to eliminate the inflow of its own arterial blood. The venous blood supply was gradually replaced by deoxygenated blood obtained by the gradual reduction of the oxygen content in the venous blood flowing through the oxygenator with a continuous inlet of nitrogen gas $\mathrm{N}_{2}$.

In the case of the ischemic model of cardiogenic shock, HF was induced by the proximal closure of one of the major branches of the left coronary artery - LAD or LCx by balloon catheter $(4 \mathrm{~mm} \times 20 \mathrm{~mm}$, or $5 \mathrm{~mm} \times 20 \mathrm{~mm}$ ) with a lumen. The first one from these two arteries that has been successfully catheterized, what was verified by radiography, was used for the protocol performance. If the HF was not successfully achieved even at low flow rates according to the protocol of $10 \mathrm{ml} / \mathrm{min}$ (no sufficient decrease of hemodynamic parameters or corresponding findings in the ECG recording) the other artery was used for insertion of the balloon catheter. The balloon position in the artery was then repeatedly verified using angiography due to the high risk of its displacement (Fig. 1).

The coronary tree distal to the catheter was hypoperfused by the hypoxic blood passing through the catheter lumen at flow rates between 80 and $10 \mathrm{ml} / \mathrm{min}$. At the beginning of hypoperfusion the flow rate behind the occlusion was set to $80 \mathrm{ml} / \mathrm{min}$ for $20 \mathrm{~min}$. Subsequently it was gradually decreased from this initial value by $10 \mathrm{ml} / \mathrm{min}$. Each newly set flow rate was stabilized for $20 \mathrm{~min}$. The flow rate was lowered 
continuously until the hemodynamic changes in terms of the defined criteria for acute HF were achieved. In addition to hemodynamic parameters ECG was recorded in order to monitor the emerging and evolving myocardial ischemia. This status was maintained for
60 min during which the development of all parameters was observed. If the decrease of the monitored heart performance parameters continued and there was an imminent risk of acute collapse, the blood flow was gently and slowly raised to the previous level.

\section{Hypoxic model}

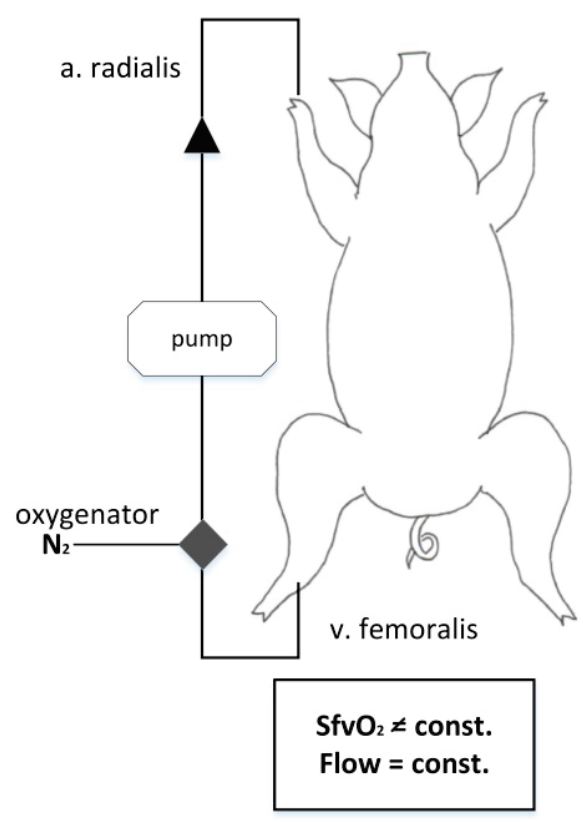

Statistics

The data are expressed as means \pm SD. ShapiroWilk normality test was used to prove the normal distribution of analyzed parameters, paired test was used. $\mathrm{P}<0.05$ was considered significant. Statistical analysis was performed using SPSS 13.0 software (SPSS Inc., Chicago, IL, USA). The difference of effects between both experimental approaches was evaluated using the chi square test for independence.

\section{Results}

The HF of the desired degree was successfully achieved in 1 animal from 5 (20\%) in the case of the hypoxic model and in 8 animals from $9(88 \%)$ in the case of the ischemic model (Table 1). This difference in an efficiency of both experimental approaches was proved to be statistically significant $(\mathrm{p}<0.001)$.

There were no defined changes in 4 animals in the hypoxic model regarding CO, LVEF, and CarFlow even in the case of coronary artery perfusion by
Ischemic model

Fig. 1. Schematic representation of the settings for both models of the heart failure. $\mathrm{N}_{2}$ - continuous inlet of nitrogen gas $\mathrm{N}_{2}$ into the oxygenator, $\mathrm{SfvO}_{2}$ - blood oxygen saturation of femoral vein (measured behind the oxygenator in the case of the hypoxic model), Flow - perfusion of the left coronary artery (controlled hypoperfusion behind the closure in the case of the ischemic model). deoxygenated blood with $\mathrm{SfvO}_{2}$ lower than $40 \%$, only $\mathrm{SvO}_{2}$ decreased below $60 \%$ in two animals. In the case of an ischemic model, only in one animal the desired decrease of any of the above defined hemodynamic parameters was not found.

In case of the ischemic model, due to interindividual differences between animals, the onset of the HF signs was achieved at different flow rates and at different times. The time that was necessary for inducing advanced failure signs vary from 18 to $142 \mathrm{~min}$ at flow rates of venous blood from 10 to $80 \mathrm{ml} / \mathrm{min}$ (Table 2). Under these conditions all 8 animals showed signs of severe acute heart failure. $\mathrm{CO}$ decreased from baseline values $6.70 \pm 0.89 \mathrm{l} / \mathrm{min}$ to $2.89 \pm 0.75 \mathrm{l} / \mathrm{min} \quad(\mathrm{P} \leq 0.001)$. This was accompanied by SV decrease from $69 \pm 33 \mathrm{ml}$ to $39 \pm 21 \mathrm{ml}(\mathrm{P} \leq 0.01)$. Also values of $\mathrm{MAP}, \mathrm{SvO}_{2}, \mathrm{CarFlow}$, and LVEF decreased significantly (Table 1, Fig. 2). The decrease of values from the Baseline to the HF was in the case of these evaluated parameters on a significance level of 0.001 . 
Table 1. Hemodynamic parameters and left ventricular performance parameters at baseline and at development of heart failure in the case of the ischemic and hypoxic models. The difference in an efficiency of both experimental approaches was proved to be statistically significant $(p<0.001)$.

\begin{tabular}{|c|c|c|c|c|c|}
\hline Parameter & Model & $\mathbf{n}$ & Baseline & HF & $\mathbf{p}$ \\
\hline \multirow{2}{*}{$C O, l / m i n$} & $\mathrm{H}$ & 1 & 4.55 & 1.73 & \\
\hline & $\mathbf{I}$ & 8 & $6.70 \pm 0.89$ & $2.89 \pm 0.75$ & $<0.001$ \\
\hline \multirow{2}{*}{$M A P, m m ~ H g$} & $\mathrm{H}$ & 1 & 116 & 48 & \\
\hline & $\mathbf{I}$ & 8 & $\mathbf{9 7} \pm \mathbf{1 7}$ & $47 \pm 6$ & $<0.001$ \\
\hline \multirow{2}{*}{$L V E F, \%$} & $\mathrm{H}$ & 1 & 52 & 20 & \\
\hline & $\mathbf{I}$ & 8 & $50 \pm 8$ & $19 \pm 2$ & $<0.001$ \\
\hline \multirow{2}{*}{$\mathrm{SvO}_{2}, \%$} & $\mathrm{H}$ & 1 & 86 & 59 & \\
\hline & $\mathbf{I}$ & 8 & $83 \pm 2$ & $58 \pm 8$ & $<0.001$ \\
\hline \multirow{2}{*}{ CarFlow, $\mathrm{ml} / \mathrm{min}$} & $\mathrm{H}$ & 1 & 310 & 89 & \\
\hline & I & 8 & $337 \pm 78$ & $136 \pm 59$ & $<0.001$ \\
\hline
\end{tabular}

Data expressed as mean \pm SD. $\mathrm{H}$ - hypoxic model, developed heart failure, $\mathrm{I}$ - ischemic model, developed heart failure, $\mathrm{n}$ - number of samples, Baseline - baseline period of no intervention, $\mathrm{HF}$ - heart failure, CO - cardiac output, MAP - mean arterial pressure, LVEF left ventricular ejection fraction, $\mathrm{SvO}_{2}$ - mixed venous blood oxygen saturation, CarFlow - blood flow in the carotid artery.

Table 2. Flow rates and times to induce advanced failure signs at the LAD or LCx branch closures which developed in 8 animals of the ischemic model with no acute mortality.

\begin{tabular}{lcccccccc}
\hline Biomodel number & $\mathbf{1}$ & $\mathbf{2}$ & $\mathbf{3}$ & $\mathbf{4}$ & $\mathbf{5}$ & $\mathbf{6}$ & $\mathbf{7}$ & $\mathbf{8}$ \\
\hline Time, min & 142 & 87 & 122 & 44 & 61 & 121 & 18 & 141 \\
Flow rate, $\mathrm{ml} / \mathrm{min}$ & 10 & 40 & 20 & 60 & 50 & 20 & 80 & 10 \\
Branch closures & Lcx & Lcx & LAD & LAD & LAD & LAD & LAD & Lcx \\
\hline
\end{tabular}

Time - time to achieve heart failure, Flow rate - flow rate behind the closure in the case of the ischemic model to achieve heart failure, Branch closures - branch of the left coronary artery where failure occurred.

\section{Discussion}

The aim of our study was an achievement of severe heart failure at very low mortality by using controlled blood desaturation or controlled hypoperfusion. A new approach in our study was the search for the optimal point for stable severe heart failure with respect to prevention of sudden circulatory arrest. The aim was not to achieve just the ischemia but to achieve a severe degree of the heart failure.

In the hypoxic model a sufficient drop in heart function parameters was not achieved even in cases when hemoglobin saturation of prefunding blood in the left coronary artery was significantly reduced. Therefore, it can be concluded that a healthy heart is able to extract enough oxygen to preserve myocardial function at rest, even if the blood is significantly desaturated, and a combination of ischemia with hypoxia is needed for the development of severe HF. Quite different situation can be observed if global hypoxia under controlled hypoventilation is used. Such situation leads to the development of really severe HF probably due to the combined effect of hypoxia and products of hypoxic metabolism (Ostadal et al. 2016).

The ischemic model is able to simulate acute cardiogenic shock secondary to acute myocardial ischemia in human medicine and represents fully shock-induced damages to healthy myocardium with symptoms of severe decompensation. 

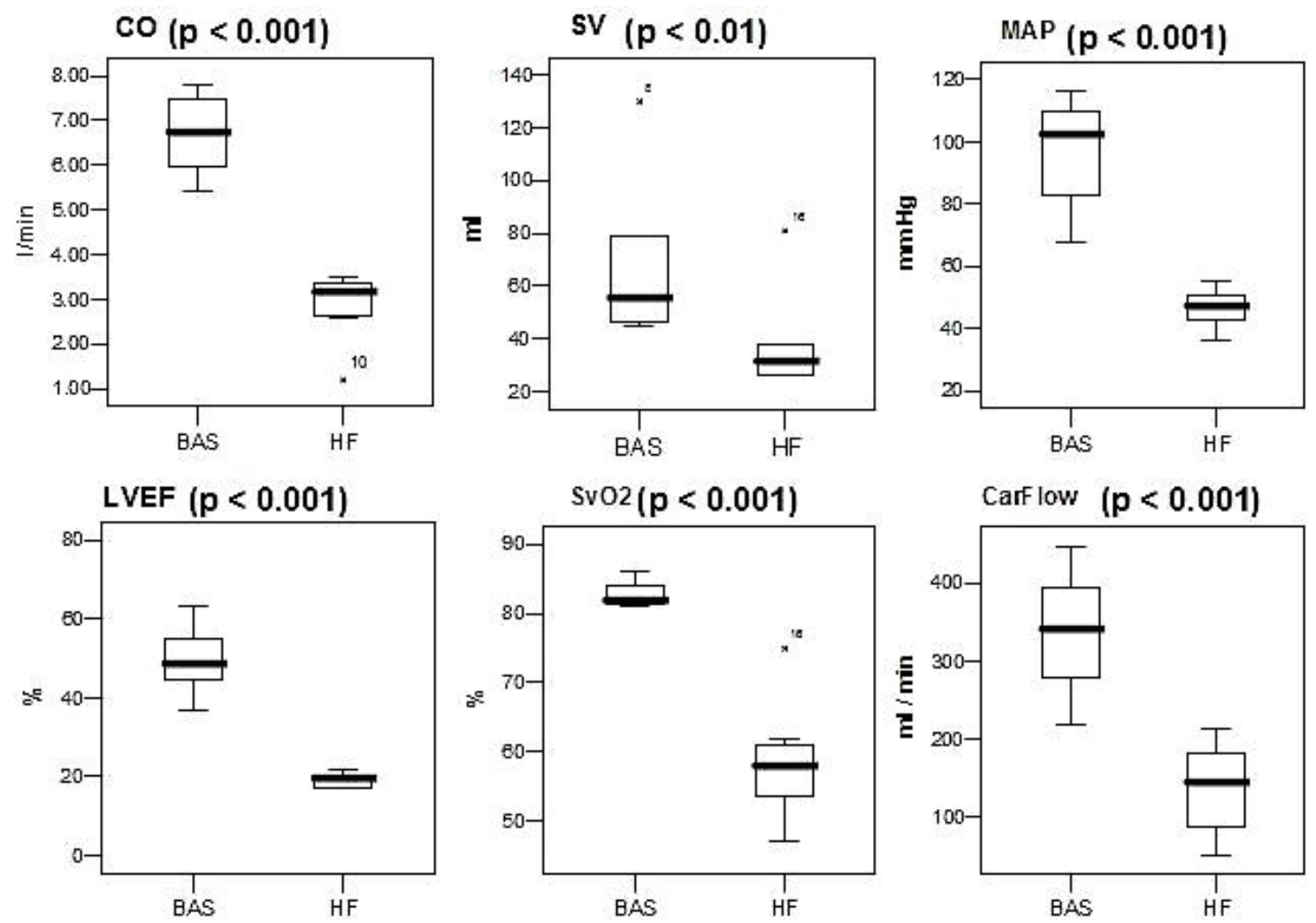

Fig. 2. Decreases in all hemodynamic parameters after development of heart failure in the ischemic model. BAS - baseline, HF - heart failure, $\mathrm{CO}$ - cardiac output, MAP - mean arterial pressure, LVEF - left ventricular ejection fraction, $\mathrm{SvO}_{2}$ - mixed venous blood saturation, CarFlow - flow in the right carotid artery, SV - stroke volume. Box-plot graphs: inside the box there are $50 \%$ of values. Thick line represents median. Top $25 \%$ of values are above the box. Bottom $25 \%$ of values are below the box as indicated by vertical lines.

In our ischemic model, the severe HF occurred in most cases after one hour, but the time varied according to the coronary artery which was occluded, what corresponded to the different efficacy of LAD and LCx in developing the HF. Occluding the proximal LAD in pigs induces larger infarct size and, depressed systolic function than occluding the LCx. Location of myocardial infarction significantly impacts the severity of $\mathrm{HF}$ (Ishikawa et al. 2014). Moreover, differences in location of LAD occlusion in swine models may affect the development of acute myocardial infarction ( $\mathrm{Li}$ et al. 2014). This fact corresponds to our finding that lower flow rates and longer time are needed for development of the HF in the case of the LCx occlusion (Table 2). Another interesting result of our study was that in individual animals different decrease of coronary blood flow was needed to achieve the HF what did not correlate with any monitored parameter. It was particularly interesting in the case of the LVEF which is the simplest and the most frequently clinically used cardiac contractility parameter (Ishikawa et al. 2012).

Another observation was that rapid reperfusion following the occlusion-balloon deflation led to severe dysrhythmias and always to ventricular fibrillation (VF), regardless of whether the heart failure was achieved or not. During the gradual development of the HF no compensation was experienced in terms of increased flow to the brain as the blood flow via carotid artery decreased. The reduction of the pumping function of the left ventricle leads to the development of other compensatory mechanisms. This further influences the reflexes regulating the blood circulation throughout the body, even at the level of the myocardium. It is questionable what is an extent of this effect and what is a level of a contribution of the overall body hypoxia depth to the central and peripheral reflexes controlling the circulation.

None of the animals under the ischemic model suffered any episode of sudden cardiac arrest during the protocol or after the steady state was achieved. Only VF episodes occurred in the event of a sudden accidental interruption of the blood flow in a coronary artery or during an abrupt resumption of full flow via the artery (>80 $\mathrm{ml} / \mathrm{min})$.

The main limitation of the presented ischemic 
model is its great variability in the coronary artery anatomy in individual animals. Repeated skiagraphy examinations are required to verify the correct position of the catheter since the outcomes are extremely sensitive to the occlusion balloon malposition or accidental extraction from the coronary arteries. For further examination it will be necessary to assess the inter-individual variation in ischemic size.

Another limitation is an impossibility to introduce a model of an acute HF when a loss of the heart pump performance occurs suddenly from a full health. In clinical medicine compromised myocardium of varying durations as well as possible comorbidities represent the most frequent scenarios. In acute model neurohumoral and compensatory mechanisms that are activated during a period of myocardial disease progression cannot be comprehensively manifested. The model of chronic heart failure provides better conditions really corresponding to a clinical medicine in which decompensation develops on the basis of the present heart damage (Hala et al. 2016).

The last limitation of the study is a small number of animals used in the experiment. Moreover, the results obtained in our study could be theoretically influenced by induced insufficiency of the aortic valve due to the insertion of transaortic catheter, but according to echocardiography findings there is no significant regurgitation across aortic valve so this limitation can be excluded.

In conclusion, the ischemic model offers a pathophysiologically accurate, technically simple and reproducible method of the heart failure induction with minimal mortality. Episodes of ventricular fibrillation did not occur during slow progressive ischemization, which is otherwise regularly accompanied by complete coronary closure with a significant increase in mortality. Individual differences in response to the different coronary artery closures, as well the different levels of perfusion needed to induce failure are likely due to the variability in the anatomy of the coronary arteries.

\section{Conflict of Interest}

There is no conflict of interest.

\section{Acknowledgements}

The authors wish to gratefully acknowledge the advice and assistance of Alena Dohnalová, for help with statistical analysis, of Tereza Vavř́ková, Alena Ehrlichová and Karel Kypta for their invaluable technical support and of Jana Bortelová for her help with animal care. This work was supported by Charles University research grants GA UK No. 662214, GAUK No. 538216, and SVV 260379.

\section{Abbreviations}

ACT - activated clotting time, CarFlow - blood flow in carotid artery, CO - cardiac output, CVP - central venous pressure, EDV - end-diastolic volume, ESV - endsystolic volume, etCO $-\mathrm{CO}_{2}$ levels at the end of exhalation, HF - heart failure, HR - heart rate, LAD left anterior descending artery, LCx - left circumflex artery, LVEF - left ventricular ejection fraction, MAP mean arterial pressure, the case of hypoxic model just behind the oxygenator, $\mathrm{SfvO}_{2}$ - blood oxygen saturation from femoral vein, in $\mathrm{SpO}_{2}$ - peripheral blood oxygen saturation, $\mathrm{SV}$ - stroke volume, $\mathrm{SvO}_{2}-$ mixed venous blood saturation, VA ECMO - venoarterial extracorporeal membrane oxygenation, VF - ventricular fibrilation.

\section{References}

CRICK S, SHEPPARD M, HO S, GEBSTEIN L, ANDERSON R: Anatomy of the pig heart: comparisons with normal human cardiac structure. J Anat 193: 105-119, 1998.

HALA P, MLCEK M, OSTADAL P, JANAK D, POPKOVA M, BOUCEK T, LACKO S, KUDLICKA J, NEUZIL P, KITTNAR O: Regional tissue oximetry reflects changes in arterial flow in porcine chronic heart failure treated with venoarterial extracorporeal membrane oxygenation. Physiol Res 65 (Suppl 5): S621-S631, 2016.

HUGHES HC: Swine in cardiovascular research. Lab Anim Sci 36: 348-350, 1986.

FUHRMAN BP, HERNAN LJ, ROTTA AT, HEARD CM, ROSENKRANZ ER: Pathophysiology of cardiac extracorporeal membrane oxygenation. Artif Organs 23: 966-969, 1999.

ISHIKAWA K, AGUERO J, TILEMANN L, LADAGE D, HAMMOUDI N, KAWASE Y, SANTOS-GALLEGO CG, FISH K, LEVINE RA, HAJJAR RJ: Characterizing preclinical models of ischemic heart failure: differences between LAD and LCx infarctions. Am J Physiol Heart Circ Physiol 307: H1478-H1486, 2014. 
ISHIKAWA K, CHEMALY ER, TILEMANN L, FISH K, LADAGE D, AGUERO J, VAHL T, SANTOS-GALLEGO C, KAWASE Y, HAJJAR RJ: Assessing left ventricular systolic dysfunction after myocardial infarction: are ejection fraction and dP/dtmax complementary or redundant? Am J Physiol Heart Circ Physiol 302: H1423-H1428, 2012.

KIM W, JEONG MH, SIM DS, HONG YJ, SONG HC, PARK JT, AHN YK: A porcine model of ischemic heart failure produced by intracoronary injection of ethyl alcohol. Heart Vessels 26: 342-348, 2011.

LI X, SHAO D, WANG G, JIANG T, WU H, GU B, CAO K, ZHANG J, QI L, CHEN Y: Effects of different LAD-blocked sites on the development of acute myocardial infarction and malignant arrhythmia in a swine model. J Thorac Dis 6: 1271-1277, 2014.

MLCEK M, OSTADAL P, BELOHLAVEK J, HAVRANEK S, HRACHOVINA M, HUPTYCH M, HALA P, HRACHOVINA V, NEUZIL P, KITTNAR O: Hemodynamic and metabolic parameters during prolonged cardiac arrest and reperfusion by extracorporeal circulation. Physiol Res 61 (Suppl 2): S57-S65, 2012.

OSTADAL P, MLCEK M, KRUGER A, HALA P, LACKO S, MATES M, VONDRAKOVA D, SVOBODA T, HRACHOVINA M, JANOTKA M, PSOTOVA H, STRUNINA S, KITTNAR O, NEUZIL P: Increasing venoarterial extracorporeal membrane oxygenation flow negatively affects left ventricular performance in a porcine model of cardiogenic shock. J Transl Med 13: 266-273, 2015.

OSTADAL P, MLCEK M, STRUNINA S, HRACHOVINA M, KRUGER A, VONDRAKOVA D, JANOTKA M, HALA P, KITTNAR O, NEUZIL P: Novel porcine model of acute severe cardiogenic shock developed by upper-body hypoxia. Physiol Res 65: 711-715, 2016.

RAGHAVAN K, WEI CL, KOTTAM A, ALTMAN DG, FERNANDEZ DJ, REYES M, VALVANO JW, FELDMAN MD, PEARCE JA: Design of instrumentation and data acquisition system for complex admittance measurement. Biomed Sci Instrum 40: 453-457, 2004.

SOLEIMANI B, PAE WE: Management of left ventricular distension during peripheral extracorporeal membrane oxygenation for cardiogenic shock. Perfusion 27: 326-331, 2012.

VARGA-SZEMES A, KISS P, BROTT BC, WANG D, SIMOR T, ELGAVISH GA: Embozene ${ }^{\mathrm{TM}}$ microspheres induced nonreperfused myocardial infarction in an experimental swine model. Catheter Cardiovasc Interv 81: 689-697, 2013. 